Project 1025342

\title{
Anaerobic Biotransformation and Mobility of Pu and PuEDTA
}

\author{
Xun, Luying \\ Washington State University
}

\begin{abstract}
RESULTS TO DATE: Objective: Isolate anaerobic EDTA-degrading bacteria.
\end{abstract}
Rational: Although our goal is to isolate anaerobic EDTA degraders, we initiated the experiments to include nitrilotriacetate (NTA), which is a structure homologue of EDTA. All the aerobic EDTA degraders can degrade NTA, but the isolated NTA degraders cannot degrade EDTA. Since NTA is a simpler structure homologue, it is likely that EDTA-degrading ability is evolved from NTA degradation. This hypothesis is further supported from our characterization of EDTA and NTA-degrading enzymes and genes (J. Bact. 179:1112-1116; and Appl. Environ. Microbiol. 67:688-695). The EDTA monooxygenase and NTA monooxygenase are highly homologous. EDTA monooxygenase can use both EDTA and NTA as substrates, but NTA monooxygenase can only use NTA as a substrate. Thus, we put our effort to isolate both NTA and EDTA degraders. In case, an anaerobic EDTA degrader is not immediately enriched, we will try to evolve the NTA degraders to use EDTA. Both aerobic and anaerobic enrichment cultures were set.

Experiments: Samples used for enrichment of NTA and EDTA degrading microorganisms have been collected from several sites. Aerobic and Anaerobic sludge from the Pullman wastewater treatment plant, as well as multiple samples from the Palouse and Snake rivers in Washington state, have been used as inoculums. We used enrichment media based on the published works of Nortemann (App. Environ. Microbiol. 58, 671-676) for aerobic EDTA degraders and Egli et al. (System. Appl. Microbiol. 10, 297-305) for anaerobic NTA degraders with nitrate as the electron acceptor. In all case, EDTA or NTA is used as the sole carbon and energy source. For anaerobic enrichment, nitrate was used as the terminal electron acceptor so that its concentration in the medium is proportionally increased than aerobic enrichment cultures. All cultures are inoculated with $2 \%$ of sample and grown at $300 \mathrm{C}$ with shaking at 200rpm for aerobic cultures. The anaerobic enrichment cultures grow very slowly. We are still enriching EDTA and NTA degraders under anaerobic conditions.

From aerobic cultures, we have isolated five bacteria (AS, N1 and N2 are from aerobic waster water sludge and $Y$ and ST are from Palouse river sediment) capable to utilize NTA as the sole carbon/energy source. The bacteria readily grow utilizing NTA and train N1 reached the highest growth yield on NTA with a final turbidity of 4 at $600 \mathrm{~nm}$. No aerobic EDTA degrading bacteria have thus far been successfully isolated. Identification of the 5 isolated NTA degrading bacteria was done by analysis of a partial $16 \mathrm{~S}$ rRNA gene sequence. Bacterial genomic DNA was first isolated and then the 16S rRNA gene amplified and sequenced. Database analysis on the 16S rRNA gene sequence using the NCBI BLAST program identified the five new isolates closely related to the following known bacteria; isolate $Y$ related to Microbacterium oxydans strain S28n, AS related to Aminobacter sp. I-Z1, N2 is related to Achromobacter sp. LMG5430, N1 is related to Microbacterium oxydans strain 187, and ST is related to Aminobacter sp. C4. Aminobacter spp. are gram negative, and they must be close relative of the NTA-degrading Aminobacter aminovorans (formerly Chelatobacter heintzii) ATCC 29600 . However, there is no previous report on NTA-degrading Microbacterium spp. M. oxydans strains are gram positive.

Moe enrichment cultures have been set up to enrich anaerobic EDTA and NTA-degrading bacteria with samples from lake, river, and stream sediments, collected on various trips.

DELIVERABLES: This is a new project and we have just started working on it.

COLLABORATIONS: L. Xun (this application) - responsible for Task 3 - determination of how electron acceptors influence anaerobic EDTA biodegradation. $\mathrm{H}$. Bolton (Project PI) - Program Management and 
Task 2 - Determine the interactions between dissimilatory metal reducing microorganisms and $\mathrm{Pu}(\mathrm{IV}) \mathrm{O} 2$ (am) and Pu(IV)-EDTA. D. Rai - Task 1 - Identify the dominant Pu-EDTA species responsible for Pu transport in the subsurface. 